\title{
Tradução
}

\section{Sobre os Corpos Flutuantes (segunda parte)}

\author{
On Floating Bodies (second part)
}

\author{
ANDRÉ KOCH TORRES ASSIS \\ Universidade Estadual de Campinas | Unicamp \\ NIVALDO BENEDITO FERREIRA CAMPOS \\ Universidade Estadual de Campinas | Unicamp
}

RESUMO Esta é a tradução da segunda e última parte do texto de Arquimedes sobre os corpos flutuantes. A primeira parte deste texto está publicada na Revista da Sociedade Brasileira de História da Ciência, v. 16, p. 69-80, 1996. Nesta segunda parte, Arquimedes discute as condições de equilíbrio para um paraboloide de revolução flutuando em um líquido.

Palavras-chave Arquimedes - centro de gravidade - corpos flutuantes.

\begin{abstract}
This is the Portuguese translation of the second and last part of Archimedes's text on floating bodies. The first part of this text has been published in Revista da Sociedade Brasileira de História da Ciência, v. 16, p. 69-80, 1996. In this second part Archimedes discusses the conditions of equilibrium of a paraboloid of revolution floating in a liquid.
\end{abstract}

Keywords Archimedes - center of gravity - floating bodies.

\section{Introdução}

Apresentamos, neste artigo, a tradução da segunda parte do texto de Arquimedes (287-212 a.C.) sobre os corpos flutuantes. A primeira parte foi publicada em 1996. ${ }^{1}$ Nesta segunda parte, Arquimedes discute as condições de equilíbrio para um paraboloide de revolução flutuando em um líquido. Esta tradução foi feita a partir da tradução em inglês dos trabalhos de Arquimedes realizada por T. L. Heath. ${ }^{2}$ Os trechos entre colchetes na tradução são de Heath. As notas de rodapé de Heath são indicadas por [N. H.]. 


\section{Tradução}

\section{Sobre os Corpos Flutuantes (segunda parte) Arquimedes}

\section{Proposição 1}

Se um sólido mais leve que um fluido está em repouso nele, o peso do sólido estará para aquele do mesmo volume do fluido como a parte imersa do sólido está para o todo.

Seja $(A+B)$ o sólido e $B$ a porção imersa no fluido.

Seja $(C+D)$ um volume igual do fluido, $C$ sendo igual em volume a $A$, e $B$ [igual] a $D$.

Suponha ainda que a linha $E$ represente o peso do sólido $(A+B),(F+G)$ represente o peso de $(C+D)$ e $G 0$ [peso] de $D$.

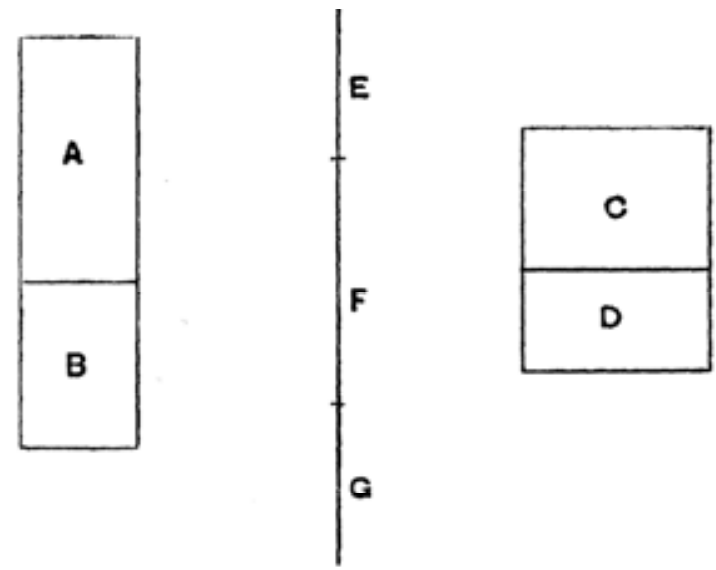

Então,

peso de $(A+B)$ : peso de $(C+D)=E:(F+G) \ldots(1)$.

E o peso de $(A+B)$ é igual ao peso de um volume $B$ do fluido [l.5], isto é, ao peso de $D$.

Quer dizer, $E=G$.

Portanto, por (1),

peso de $(A+B):$ peso de $(C+D)=G: F+G$

$$
\begin{aligned}
& =D: C+D \\
& =B: A+B .
\end{aligned}
$$

\section{Proposição 2}

Se um segmento reto de um paraboloide de revolução cujo eixo não é maior que que (3/4)p (onde p é o parâmetro principal da parábola geradora), e cuja gravidade específica é menor que a de um fluido, for colocado no fluido com seu eixo inclinado em relação à vertical por um ângulo qualquer, mas de tal forma que a base do segmento não toque a superfície do fluido, o segmento do paraboloide não permanecerá nesta posição, mas retornará à posição na qual seu eixo é vertical. 
Seja $A N$ o eixo do segmento do paraboloide e através de $A N$ trace um plano perpendicular à superfície do fluido. Faça o plano intersectar o paraboloide na parábola $B A B^{\prime}$, a base do segmento do paraboloide em $B B^{\prime}$ e 0 plano da superfície do fluido na corda $00^{\prime}$ da parábola.

Então, uma vez que o eixo $A N$ é colocado em uma posição não perpendicular em relação a $Q 0^{\prime}, B B^{\prime}$ não será paralelo a $00^{\prime}$.

Trace a tangente $P T$ à parábola, [sendo esta tangente] paralela a $Q 0^{\prime}$, e seja $P$ o ponto de contato. ${ }^{1}$

[A partir de $P$ trace $P V$ paralelo a $A N$ encontrando $00^{`}$ em $V$. Então, $P V$ será um diâmetro da parábola e também 0 eixo da porção do paraboloide imerso no fluido.

Seja $C$ o centro de gravidade do paraboloide $B A B^{\prime}$ e $F$ o [centro de gravidade] da porção imersa no fluido. Una $F C$ e 0 estenda até $H$ de forma que $H$ seja o centro de gravidade da porção restante do paraboloide acima da superfície.

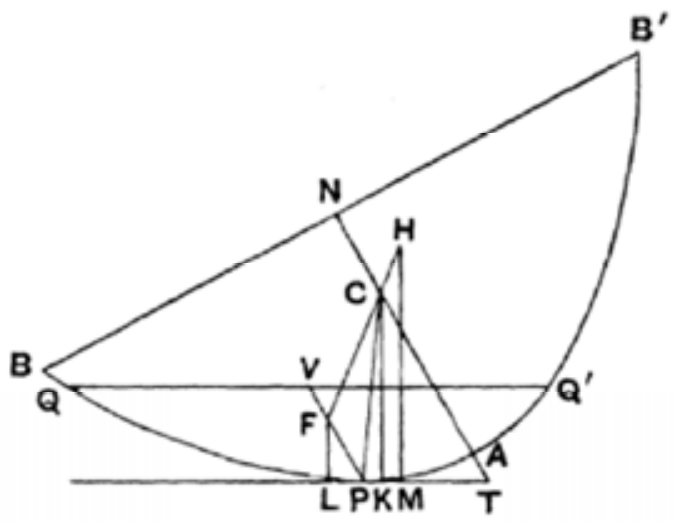

Então, uma vez que ${ }^{2}$

$$
A N=\frac{3}{2} A C
$$

e

$$
A N \ngtr \frac{3}{4} p,
$$

segue que

$$
A C \ngtr \frac{p}{2} .
$$

Portanto, se $C P$ for ligado, o ângulo CPT é agudo. ${ }^{3}$ Portanto, se $C K$ for traçado perpendicular a $P T$, $K$ estará entre $P$ e $T$. E, se $F L$ e $H M$ forem traçados paralelos a $C K$ para encontrar $P T$, cada um deles será perpendicular à superfície do fluido.

Agora, a força atuando na porção imersa do segmento do paraboloide irá atuar para cima ao longo de $L F$, enquanto o peso da porção fora do fluido atuará para baixo ao longo de $H M$.

Portanto, não haverá equilíbrio, mas o segmento irá girar de forma que $B$ se elevará e $B^{\prime}$ irá descer, até que $A N$ assuma a posição vertical.]

1 [N. H.] 0 restante da prova está faltando na versão de Tartaglia, mas é fornecido entre colchetes como apresentado por Commandinus.

2 [N. H.] Como a determinação do centro de gravidade de um segmento de um paraboloide que é assumida aqui não aparece em qualquer trabalho existente de Arquimedes, ou em qualquer trabalho conhecido de qualquer outro matemático grego, parece provável que esta determinação tenha sido investigada pelo próprio Arquimedes em algum tratado que hoje em dia está perdido.

3 [N. H.] A verdade desta afirmativa é facilmente provada a partir da propriedade da subnormal. Pois, se a normal em $P$ encontra o eixo em $G$, então $A G$ é maior do que $\frac{p}{2}$, exceto no caso em que a normal é a normal no próprio vértice $A$. Mas este último caso está excluído aqui pois, por hipótese, $A N$ não é colocada verticalmente. Portanto, sendo $P$ um ponto diferente de $A$, vem que $A G$ será sempre maior do que $A C$; e, como é reto o ângulo $T P G$, o ângulo TPC tem de ser agudo. 
[Com o propósito de comparação, serão acrescentados os equivalentes trigonométricos desta e de outras proposições.

Suponha que o ângulo NTP, no qual, na figura acima, o eixo AN é inclinado em relação à superfície do fluido, seja denotado por $\theta$.

Então as coordenadas de $P$ referidas a $A N$ e à tangente em $A$ como eixos são

onde $p$ é o parâmetro principal.

$$
\frac{p}{4} \cot ^{2} \theta, \quad \frac{p}{2} \cot \theta
$$

Suponha que $A N=h$ e que $P V=k$.

Se agora $x^{\prime}$ for a distância, a partir de $T$, da projeção ortogonal de $F$ sobre $T P$, e $x$ for a distância correspondente para o ponto $C$, temos

$$
\begin{aligned}
x^{\prime}= & \frac{p}{2} \cot ^{2} \theta \cdot \cos \theta+\frac{p}{2} \cot \theta \cdot \operatorname{sen} \theta+\frac{2}{3} k \cos \theta, \\
x= & \frac{p}{4} \cot ^{2} \theta \cdot \cos \theta+\frac{2}{3} h \cos \theta, \\
& x^{\prime}-x=\cos \theta\left\{\frac{p}{4}\left(\cot ^{2} \theta+2\right)-\frac{2}{3}(h-k)\right\} .
\end{aligned}
$$

de onde vem

A fim de que o segmento do paraboloide possa girar na direção que aumenta o ângulo PTN, $x$ ' precisa ser maior que $x$, ou seja, a expressão encontrada precisa ser positiva.

Este será sempre o caso, seja qual for o valor de $\theta$, se

ou

$$
\begin{aligned}
& \frac{p}{2} \nless \frac{2 h}{3} . \\
& \left.h \ngtr \frac{3}{4} p .\right]
\end{aligned}
$$

\section{Proposição 3}

Se um segmento reto de um paraboloide de revolução cujo eixo não é maior que que (3/4)p (onde p é o parâmetro), e cuja gravidade específica é menor que a de um fluido, for colocado no fluido com seu eixo inclinado de qualquer ângulo com a vertical, mas de forma que sua base esteja inteiramente submersa, o sólido não permanecerá nesta posição, mas retornará à posição na qual o eixo é vertical.

Seja $A N$ o eixo do paraboloide e através de $A N$ trace um plano perpendicular à superfície do fluido, interseccionando o paraboloide na parábola $B A B^{\prime}$, a base do segmento em $B N B^{\prime}$ e o plano da superfície do fluido na corda $00^{\prime}$ da parábola.

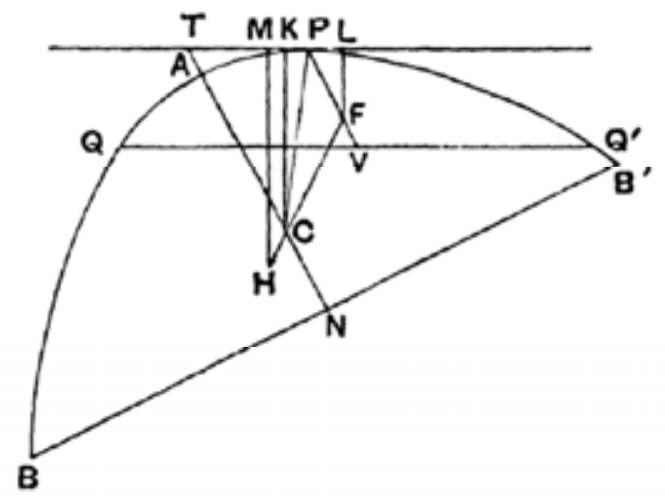


Então, uma vez que $A N$, como colocado, não é perpendicular à superfície do fluido, $00^{\prime}$ e $B B^{\prime}$ não serão paralelos.

Trace $P T$ paralelo a $00^{\prime}$ e tocando a parábola em $P$. Faça $P T$ encontrar o prolongamento de $N A$ em $T$. Trace 0 diâmetro $P V$ dividindo ao meio $00^{\prime}$ em $V$. Então, $P V$ é o eixo da porção do paraboloide acima da superfície do fluido.

Seja $C$ o centro de gravidade do segmento completo do paraboloide e $F$ [o centro de gravidade] da porção acima da superfície. Una $F C$ e o prolongue até $H$, de forma que $H$ seja o centro de gravidade da porção imersa.

Então, uma vez que $A C \ngtr \frac{p}{2}, 0$ ângulo $C P T$ é um ângulo agudo, como na proposição anterior.

Portanto, se $C K$ for traçado perpendicular a $P T, K$ estará entre $P$ e $T$. Também, se $H M$ e $F L$ forem traçados paralelos a $C K$, eles serão perpendiculares à superfície do fluido.

E a força atuando na porção submersa atuará para cima, ao longo de $H M$, enquanto o peso do restante atuará para baixo ao longo do prolongamento de $L F$.

Desta forma o paraboloide girará até que assuma a posição na qual AN seja vertical.

\section{Proposição 4}

Dado um segmento reto de um paraboloide de revolução cujo eixo AN é maior que (3/4)p (onde p é o parâmetro), e cuja gravidade específica é menor que a de um fluido, mas tem em relação a ele uma razão não menor que $\left(A N-\frac{3}{4} p\right)^{2}: A N^{2}$, se o segmento do paraboloide for colocado no fluido com seu eixo inclinado de qualquer ângulo em relação à vertical, mas de forma que sua base não toque a superfície do fluido, ele não permanecerá nesta posição, mas retornará à posição na qual seu eixo seja vertical.

Seja $A N$ o eixo do segmento do paraboloide, e deixe um plano ser traçado através de $A N$ perpendicularmente à superfície do fluido e interseccionando o segmento na parábola $B A B^{\prime}$, a base do segmento em $B B^{\prime}$, e a superfície do fluido na corda $00^{\prime}$ da parábola.

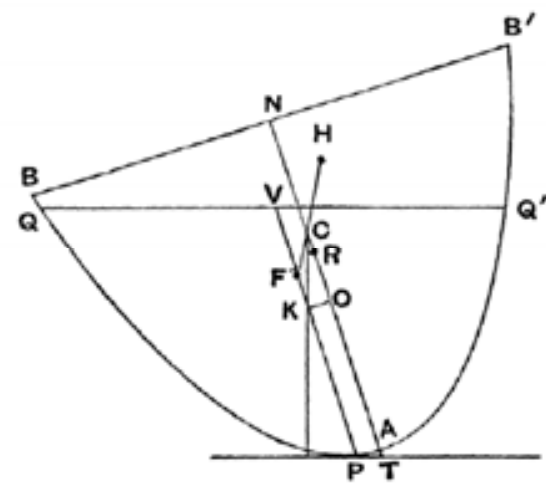

Então, $A N$, como colocado, não será perpendicular a $00^{\prime}$.

Trace $P T$ paralelo a $00^{\prime}$ e tocando a parábola em $P$. Trace o diâmetro $P V$ dividindo ao meio $00^{\prime}$ em $V$. Desta forma $P V$ será o eixo da porção submersa do sólido.

Seja $C$ o centro de gravidade do sólido completo e $F$ [0 centro de gravidade] da porção imersa. Una $F C$ e 0 prolongue até $H$, de forma que $H$ seja o centro de gravidade da porção restante.

Agora, uma vez que

$$
A N=\frac{3}{2} A C,
$$

e

$$
A N>\frac{3}{4} p
$$


segue que

$$
A C>\frac{p}{2}
$$

Meça $C O$ ao longo de $C A$ igual a $\frac{p}{2}$, e $O R$ ao longo de $O C$ igual a $\frac{1}{2} A O$.

Então, uma vez que

$$
A N=\frac{3}{2} A C
$$

e

$$
A R=\frac{3}{2} A O
$$

temos, por subtração,

$$
N R=\frac{3}{2} O C
$$

Isto é,

$\mathrm{OU}$

$$
\begin{aligned}
A N-A R & =\frac{3}{2} O C \\
& =\frac{3}{4} p,
\end{aligned}
$$

$$
A R=\left(A N-\frac{3}{4} p\right)
$$

Então,

$$
\left(A N-\frac{3}{4} p\right)^{2}: A N^{2}=A R^{2}: A N^{2},
$$

e, portanto, a razão da gravidade específica do sólido para a do fluido é, pelo enunciado, não menos que a razão $A R^{2}: A N^{2}$.

Mas, pela Prop. 1, a razão anterior é igual à razão da porção imersa para todo 0 sólido, isto é, [é igual] à razão $P V^{2}: A N^{2}$ [Sobre Conoides e Esferoides, Prop. 24].

Assim,

ou

Segue que

$$
\begin{gathered}
P V^{2}: A N^{2} \nless A R^{2}: A N^{2}, \\
P V \nless A R .
\end{gathered}
$$

$$
\begin{aligned}
P F\left(=\frac{2}{3} P V\right) & \nless \frac{2}{3} A R \\
& \nless A O .
\end{aligned}
$$

Se, portanto, $O K$ for traçado a partir de $O$ perpendicularmente em relação a $A O$, ele encontrará $P F$ entre $P$ e $F$. Também, se CK for unido, o triângulo KCO será igual e similar ao triângulo formado pela normal, pela subnormal e pela ordenada em $P$ (uma vez que $C O=\frac{1}{2} p$ ou a subnormal, e $K O$ é igual à ordenada).

Logo, $C K$ é paralelo à normal em $P$ e, portanto, perpendicular à tangente em $P$ e [perpendicular] à superfície do fluido.

Portanto, se forem traçados paralelos a $C K$ através de $F$ e de $H$, eles serão perpendiculares à superfície do fluido, e a força agindo na porção submersa do sólido atuará para cima, ao longo do primeiro, enquanto o peso da outra porção atuará para baixo ao longo do segundo.

Portanto o sólido não permanecerá em sua posição, mas irá girar até que $A N$ assuma uma posição vertical.

[Usando a mesma notação de antes (nota seguindo a Prop. 2), temos

$$
x^{\prime}-x=\cos \theta\left\{\frac{p}{4}\left(\cot ^{2} \theta+2\right)-\frac{2}{3}(h-k)\right\} \text {, }
$$


e o valor mínimo da expressão dentro das chaves, para diferentes valores de $\theta$, é

$$
\frac{p}{2}-\frac{2}{3}(h-k)
$$

correspondendo à posição na qual $A M$ é vertical, ou $\theta=\frac{\pi}{2}$. Portanto, somente haverá equilíbrio estável nesta posição, se

$$
k \nless\left(h-\frac{3}{4} p\right),
$$

ou, se $s$ for a razão da gravidade específica do sólido para a do fluido $\left(=k^{2} / h^{2}\right.$ neste caso),

$$
s \nless\left(h-\frac{3}{4} p\right)^{2} / h^{2} \text {.] }
$$

\section{Proposição 5}

Dado um segmento reto de um paraboloide de revolução cujo eixo AN é maior que (3/4)p e (onde p é o parâmetro), e sua gravidade específica é menor que a de um fluido, mas em uma razão para ela não maior do que a razão $\left\{A N^{2}-\left(A N-\frac{3}{4} p\right)^{2}\right\}: A N^{2}$, se o segmento for colocado no fluido com seu eixo inclinado de qualquer ângulo em relação à vertical, mas de forma que sua base esteja completamente submersa, ele não permanecerá nesta posição, mas retornará à posição na qual AN é vertical.

Seja um plano traçado por $A N$, como colocado, perpendicular à superfície do fluido e cortando o segmento do paraboloide na parábola $B A B^{\prime}$, a base do segmento em $B B^{\prime}$, e o plano da superfície do fluido na corda $Q Q^{\prime}$ da parábola.

Trace a tangente $P T$ paralela a $Q Q^{\prime}$ e o diâmetro $P V$, dividindo ao meio $Q Q^{\prime}$, será consequentemente 0 eixo da porção do paraboloide acima da superfície do fluido.

Seja $F$ o centro de gravidade da porção acima da superfície, $C$ [0 centro de gravidade] de todo o sólido, e prolongue $F C$ até $H$, o centro de gravidade da porção imersa.

Como na última proposição, $A C>\frac{p}{2}$, e medimos $C O$ ao longo de $C A$ igual a $\frac{p}{2}$, e $O R$ ao longo de $O C$ igual a $\frac{1}{2} A O$

Então, $A N=\frac{3}{2} A C$ e $A R=\frac{3}{2} A O$;

e derivamos, como antes,

$$
A R=\left(A N-\frac{3}{4} p\right)
$$

Agora, por hipótese,

(gravidade específica do sólido) : (gravidade específica do fluido)

$$
\begin{gathered}
\ngtr\left\{A N^{2}-\left(A N-\frac{3}{4} p\right)^{2}\right\}: A N^{2} \\
\ngtr\left(A N^{2}-A R^{2}\right): A N^{2} .
\end{gathered}
$$




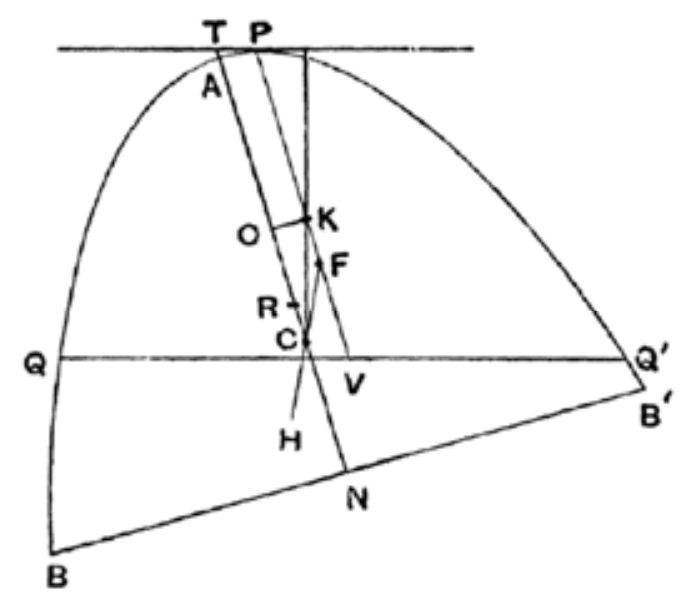

Portanto,

e

Assim,

do que segue que

e

(porção submersa) : (todo o sólido) $\ngtr\left(A N^{2}-A R^{2}\right): A N^{2}$, (todo o sólido) : (porção acima da superfície) $\ngtr A N^{2}: A R^{2}$.

$$
\begin{gathered}
A N^{2}: P V^{2} \ngtr A N^{2}: A R^{2}, \\
P V \nless A R \\
P F \nless A R \\
\nless A O .
\end{gathered}
$$

Portanto, se for traçada uma perpendicular até $A C$ a partir de 0 , ela encontrará $P F$ em algum ponto $K$ entre $P$ e $F$.

$\mathrm{E}$, uma vez que $C O=\frac{1}{2} p, C K$ será perpendicular a $P T$, como na última proposição.

Agora a força atuando na porção submersa do sólido atuará para cima através de $H$, e o peso da outra porção atuará para baixo através de $F$, em direções paralelas, em ambos os casos, a $C K$; de onde segue a proposição.

\section{Proposição 6}

Se um segmento reto de um paraboloide mais leve que um fluido for tal que seu eixo AM seja maior que (3/4)p, mas $A M: \frac{1}{2} p<15: 4$, e se o segmento for colocado no fluido com seu eixo tão inclinado em relação à vertical que sua base toque o fluido, ele nunca permanecerá em uma posição tal que a base toque a superfície somente em um ponto.

Suponha que o segmento do paraboloide seja colocado na posição descrita, e deixe o plano através do eixo $A M$ perpendicular à superfície do fluido interseccionar o segmento do paraboloide no segmento parabólico $B A B^{\prime}$ e 0 plano da superfície do fluido em $B O$.

Tome $C$ em $A M$ tal que $A C=2 C M$ (ou tal que $C$ seja o centro de gravidade do segmento do paraboloide), e meça $C K$ ao longo de $C A$ tal que

$$
A M: C K=15: 4
$$




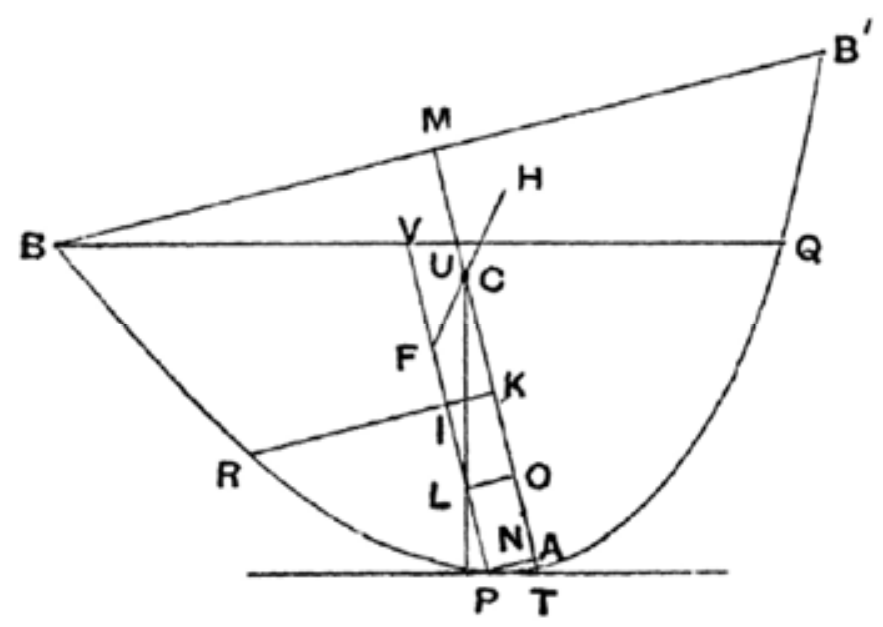

Então, $A M: C K>A M: \frac{1}{2} p$, por hipótese; portanto, $C K<\frac{1}{2} p$.

Meça $C O$ ao longo de $C A$ igual a $\frac{1}{2} p$. Também trace $K R$ perpendicular a $A C$ encontrando a parábola em $R$.

Trace a tangente $P T$ paralela a $B Q$, e através de $P$ trace o diâmetro $P V$ dividindo ao meio $B O$ em $V$ e encontrando $K R$ em $I$.

Então,

$P V: P I \geq K M: A K$

"o que já está provado". 4

4 [N. H.] Não temos nenhuma pista sobre 0 trabalho no qual a prova desta proposição estava contida. A prova a seguir é mais curta que a de Robertson (no Apêndice da edição de Torelli).

Deixe $B Q$ encontrar $A M$ em $U$, e faça $P N$ ser a ordenada a partir de $P$ até $A M$.

Temos que provar que $P V . A K \geq P I . K M$, ou em outras palavras que

$$
(P V . A K-P I . K M) \text { é positivo ou zero. }
$$

$$
\text { Agora } \quad \begin{array}{rl}
P V \cdot A K-P I . K M=A K & P V-(A K-A N)(A M-A K) \\
& =A K^{2}-A K(A M+A N-P V)+A M \cdot A N \\
& =A K^{2}-A K \cdot U M+A M \cdot A N
\end{array}
$$

(uma vez que $A N=A T)$.

Agora,

$U M: B M=N T: P N$,

Portanto,

$$
U M^{2}: p \cdot A M=4 A N^{2}: p \cdot A N,
$$

de onde vem que

$$
U M^{2}=4 A M \cdot A N,
$$

ou

$$
A M \cdot A N=\frac{U M^{2}}{4} .
$$

Portanto,

$$
P V \cdot A K-P I . K M=A K^{2}-A K \cdot U M+\frac{U M^{2}}{4}=\left(A K-\frac{U M}{2}\right)^{2},
$$

e, consequentemente, $(P V . A K-P I . K M)$ não pode ser negativo. 


$$
C K=\frac{4}{15} A M=\frac{2}{5} A C ；
$$

de onde vem que

$$
A K=A C-C K=\frac{3}{5} A C=\frac{2}{5} A M .
$$

Então,

$$
K M=\frac{3}{5} A M
$$

Portanto,

$$
K M=\frac{3}{2} A K
$$

Segue que

$$
P V \geq \frac{3}{2} P I,
$$

de forma que

$$
P I \leq 2 I V .
$$

Seja $F$ o centro de gravidade da porção imersa do paraboloide, tal que $P F=2 F V$. Prolongue $F C$ até $H$, o centro de gravidade da porção acima da superfície.

Trace OL perpendicular a $P V$.

Então, uma vez que $C O=\frac{1}{2} p, C L$ precisa ser perpendicular a $P T$ e, portanto, [perpendicular] à superfície do fluido.

E as forças atuando na porção imersa do paraboloide e a porção acima da superfície agem, respectivamente, para cima e para baixo ao longo de linhas que passam por $\mathrm{F}$ e $\mathrm{H}$ e são paralelas a $\mathrm{CL}$.

Portanto, o paraboloide não pode permanecer na posição na qual $B$ apenas toca a superfície, mas precisa girar na direção na qual o ângulo PTM aumenta.

A prova é a mesma no caso onde o ponto / não está sobre $V P$, mas sim sobre o prolongamento de $V P$, como na segunda figura. ${ }^{5}$

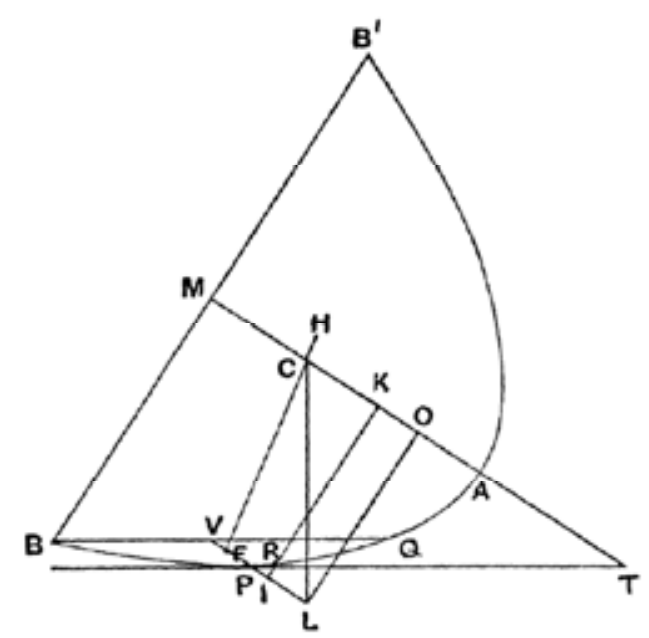

[Com a notação usada ao final da Proposição 2, se a base $B B^{\prime}$ tocar a superfície do fluido em $B$, temos

$$
B M=B V \operatorname{sen} \theta+P N,
$$

e, pela propriedade da parábola,

$$
\begin{aligned}
B V^{2} & =(p+4 A N) P V \\
& =p k\left(1+\cot ^{2} \theta\right) .
\end{aligned}
$$

5 [N. H.] É curioso que as figuras dadas por Torelli, Nizze e Heiberg são todas incorretas, uma vez que todas elas fazem o ponto o qual chamei de / situar-se sobre $B Q$, ao invés do prolongamento de VP. 
Portanto,

$$
\sqrt{p h}=\sqrt{p k}+\frac{p}{2} \cot \theta
$$

Para obter o resultado da proposição, temos que eliminar $k$ entre esta equação e

$$
x^{\prime}-x=\cos \theta\left\{\frac{p}{4}\left(\cot ^{2} \theta+2\right)-\frac{2}{3}(h-k)\right\} \text {. }
$$

Temos, da primeira equação,

$\mathrm{OU}$

$$
\begin{aligned}
& k=h-\sqrt{p h} \cot \theta+\frac{p}{4} \cot ^{2} \theta, \\
& h-k=\sqrt{p h} \cot \theta-\frac{p}{4} \cot ^{2} \theta .
\end{aligned}
$$

Portanto,

$$
\begin{aligned}
x^{\prime}-x= & \cos \theta\left\{\frac{p}{4}\left(\cot ^{2} \theta+2\right)-\frac{2}{3}\left(\sqrt{p h} \cot \theta-\frac{p}{4} \cot ^{2} \theta\right)\right\} \\
& =\cos \theta\left\{\frac{p}{4}\left(\frac{5}{3} \cot ^{2} \theta+2\right)-\frac{2}{3} \sqrt{p h} \cot \theta\right\} .
\end{aligned}
$$

Então, se o sólido não pode nunca permanecer na posição descrita, mas precisa girar na direção na qual 0 ângulo PTM aumenta, a expressão dentro das chaves precisa ser positiva seja qual for 0 valor de $\theta$.

Portanto,

$$
\begin{aligned}
\left(\frac{2}{3}\right)^{2} p h & <\frac{5}{6} p^{2} \\
h & \left.<\frac{15}{8} p .\right]
\end{aligned}
$$

\section{Proposição 7}

Dado um segmento reto de um paraboloide de revolução mais leve que um fluido e tal que seu eixo AM seja maior que (3/4)p, mas $A M: \frac{1}{2} p<15: 4$, se o segmento for colocado no fluido de forma que sua base esteja inteiramente submersa, ele nunca irá permanecer em uma posição em que a base toca a superfície do fluido somente em um ponto.

Suponha o sólido colocado de forma que somente um ponto $(B)$ da base toque a superfície do fluido. Deixe 0 plano através de $B$ e 0 eixo $A M$ cortar o sólido no segmento parabólico $B A B^{\prime}$ e o plano da superfície do fluido [cortar o sólido] na corda $B O$ da parábola.

Seja $C$ o centro de gravidade do segmento, de forma que $A C=2 C M$; e meça $C K$ ao longo de $C A$, tal que

$$
A M: C K=15: 4 \text {. }
$$

Segue que

$$
C K<\frac{1}{2} p
$$

Meça $C O$ ao longo de $C A$ igual a $\frac{1}{2} p$. Trace $K R$ perpendicular a $A M$ encontrando a parábola em $R$.

Seja $P T$, tocando (a parábola) em $P$, a tangente à parábola que é paralela a $B Q$, e seja $P V$ o diâmetro dividindo ao meio $B Q$, isto é, o eixo da porção do paraboloide acima da superfície. 


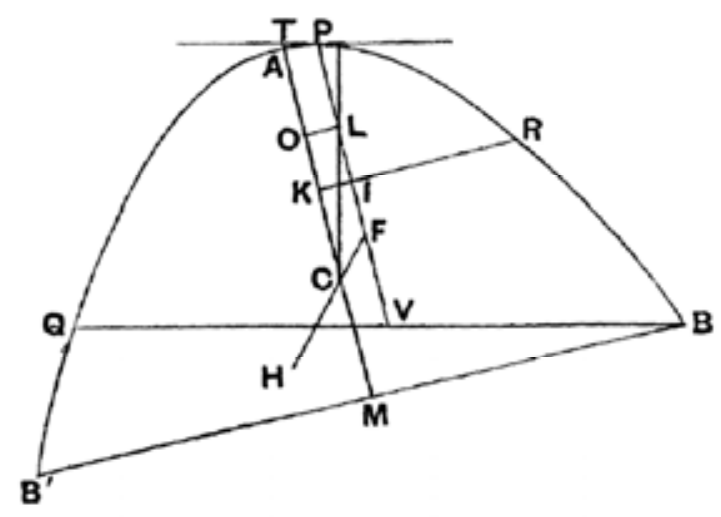

Então, como na proposição anterior, provamos que

$$
\begin{aligned}
& P V \\
\text { e } & \geq \frac{3}{2} P I, \\
P I & \leq 2 I V .
\end{aligned}
$$

Seja $F$ o centro de gravidade da porção do sólido acima da superfície; una $F C$ e o prolongue até $H$, o centro de gravidade da porção submersa.

Trace $O L$ perpendicular a $P V$; e, como antes, uma vez que $C O=\frac{1}{2} p, C L$ é perpendicular à tangente $P T$. E as linhas através de $H$ e $F$ paralelas a $C L$ são perpendiculares à superfície do fluido; portanto, a proposição é estabelecida como antes.

A prova é a mesma se o ponto / não está sobre $V P$, mas sobre o prolongamento de $V P$.

\section{Proposição 8}

Dado um sólido na forma de um segmento reto de um paraboloide de revolução, cujo eixo $A M$ é maior que (3/4)p, mas tal que $A M: \frac{1}{2} p<15: 4$, e cuja gravidade específica está para a de um fluido em uma razão menor que $\left(A M-\frac{3}{4} p\right)^{2}: A M^{2}$, então, se o sólido for colocado no fluido de forma que sua base não toque o fluido e seu eixo esteja inclinado de um ângulo com a vertical, o sólido não retornará à posição na qual seu eixo é vertical e não permanecerá em qualquer posição, exceto aquela na qual seu eixo forma com a superfície do fluido um certo ângulo a ser descrito.

Seja am tomado igual ao eixo $A M$ e seja $c$ um ponto sobre am tal que $a c=2 \mathrm{~cm}$. Meça $c o$ ao longo de ca igual a $\frac{1}{2} p$ e or ao longo de oc igual a $\frac{1}{2} a o$. 


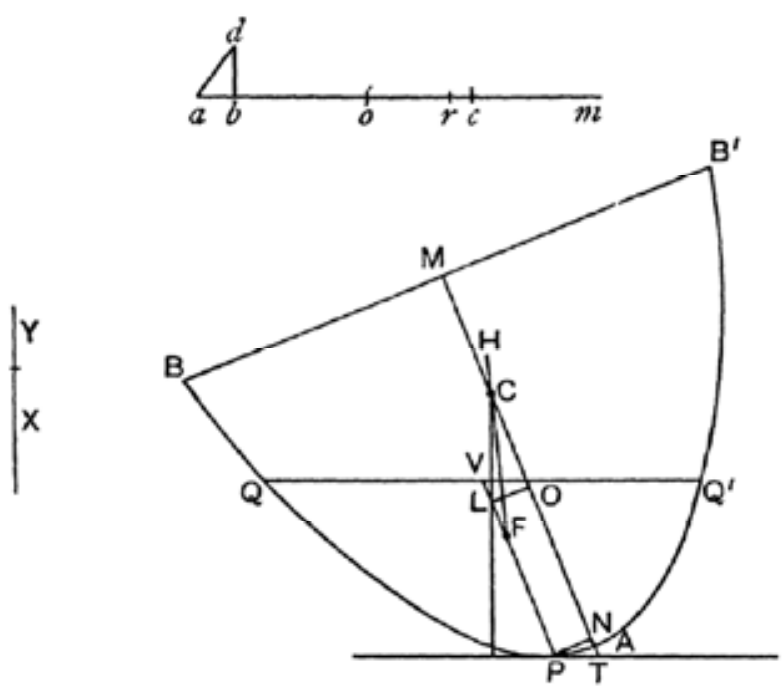

Seja $X+Y$ uma linha reta tal que (gravidade específica do sólido) : (gravidade específica do fluido) $=(X+Y)^{2}: a m^{2}$ $(\alpha)$, e suponha que $X=2 Y$.

Agora,

$$
\begin{aligned}
a r=\frac{3}{2} a o & =\frac{3}{2}\left(\frac{2}{3} a m-\frac{1}{2} p\right) \\
& =a m-\frac{3}{4} p \\
& =A M-\frac{3}{4} p .
\end{aligned}
$$

Portanto, por hipótese,

$$
(X+Y)^{2}: a m^{2}<a r^{2}: a m^{2}
$$

de onde vem que $(X+Y)<$ ar, e, portanto, $X<$ ao.

Meça $o b$ ao longo de oa igual a $X$, e trace $b d$ perpendicular a $a b$ e de comprimento tal que

$$
b d^{2}=\frac{1}{2} c o . a b
$$

Una ad.

Agora, seja o sólido colocado no fluido com seu eixo $A M$ inclinado de um ângulo com a vertical. Através de $A M$ trace um plano perpendicular à superfície do fluido, e faça este plano cortar o paraboloide na parábola $B A B^{\prime}$ e 0 plano da superfície do fluido na corda $00^{\prime}$ da parábola.

Trace a tangente $P T$ paralela a $Q 0^{\prime}$, tocando em $P$, e seja $P V$ o diâmetro dividindo ao meio $Q 0^{\prime}$ em $V$ lou 0 eixo da porção imersa do sólido), e seja $P N$ a ordenada a partir de $P$.

Meça $A O$ ao longo de $A M$ igual a ao, e meça $O C$ ao longo de $O M$ igual a oc, e trace $O L$ perpendicular a $P V$.

I. Suponha o ângulo OTP maior que o ângulo dab.

$$
\begin{aligned}
& \text { Deste modo } \\
& P N^{2}: N T^{2}>d b^{2}: b a^{2} \text {. } \\
& \text { Mas } \\
& P N^{2}: N T^{2}=p: 4 A N \\
& =c o: N T \text {, } \\
& \text { e } \\
& d b^{2}: b a^{2}=\frac{1}{2} c o: a b, \text { por }(\beta) \text {. } \\
& N T<2 a b ， \\
& \text { ou } \\
& A N<a b \text {, }
\end{aligned}
$$


de onde vem que

$$
\begin{aligned}
N O & >b o \quad(\text { uma vez que ao }=A 0) \\
& >X .
\end{aligned}
$$

Agora $(X+Y)^{2}: a m^{2}=($ gravidade específica do sólido $):($ gravidade específica do fluido $)$ $=($ porção imersa $):($ restante do sólido $)$

$$
=P V^{2}: A M^{2} \text {, }
$$

de forma que

$$
X+Y=P V \text {. }
$$

Mas

$$
P L(=N O)>X
$$

$$
\begin{aligned}
& >\frac{2}{3}(X+Y) \text {, uma vez que } X=2 Y, \\
& >\frac{2}{3} P V,
\end{aligned}
$$

ou

$$
P V<\frac{3}{2} P L
$$

e, portanto,

$$
P L>2 L V \text {. }
$$

Tome um ponto $F$ sobre $P V$ de forma que $P F=2 F V$, isto é, de forma que $F$ seja o centro de gravidade da porção imersa do sólido.

Também $A C=a c=\frac{2}{3} a m=\frac{2}{3} A M$ e, portanto, $C$ é o centro de gravidade de todo o sólido.

Una FC e o prolongue até $H$, o centro de gravidade da porção do sólido sobre a superfície.

Agora, uma vez que $C O=\frac{1}{2} p, C L$ é perpendicular à superfície do fluido; portanto, assim também são 382 [perpendiculares à superfície] as paralelas a $C L$ através de $F$ e $H$. Mas a força na porção imersa age para cima através de $F$ e aquela [força] sobre o resto do sólido [age] para baixo através de $H$.

Portanto, o sólido não ficará em repouso, mas vai girar na direção em que o ângulo MTP diminui.

II. Suponha o ângulo OTP menor que o ângulo dab. Neste caso, teremos, ao invés dos resultados acima, o seguinte,

$$
\begin{aligned}
& A N>a b, \\
& N O<X . \\
& P V>\frac{3}{2} P L, \\
& P L<2 L V .
\end{aligned}
$$

Também

e, portanto,

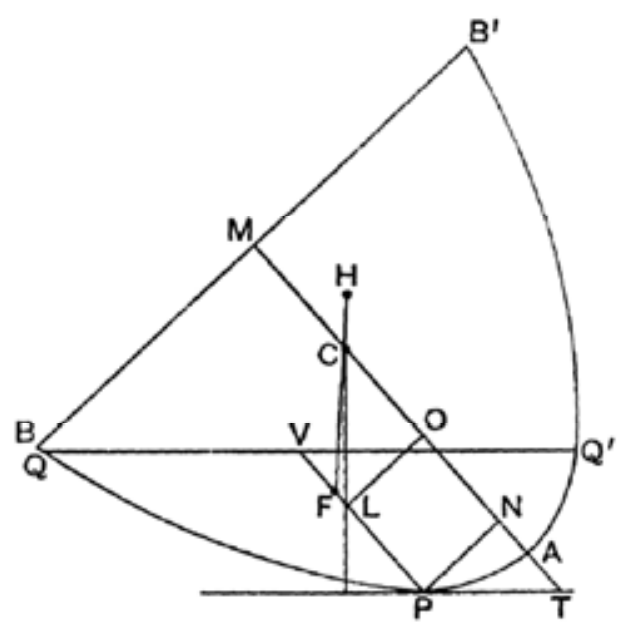


Faça $P F$ igual a $2 F V$, de forma que $F$ seja o centro de gravidade da porção imersa.

$E$, procedendo como antes, provamos neste caso que o sólido girará na direção em que o ângulo MTP aumenta.

III. Quando o ângulo MTP é igual ao ângulo dab, igualdades substituem as desigualdades nos resultados obtidos, e o próprio $L$ é o centro de gravidade da porção imersa. Assim, todas as forças agem em uma linha reta, a perpendicular $C L$; portanto, há equilíbrio e o sólido permanecerá em repouso na posição descrita.

[Com a notação usada antes

$$
x^{\prime}-x=\cos \theta\left\{\frac{p}{4}\left(\cot ^{2} \theta+2\right)-\frac{2}{3}(h-k)\right\} .
$$

e a posição de equilíbrio é obtida igualando a zero a expressão entre chaves. Temos então

$$
\frac{p}{4} \cot ^{2} \theta=\frac{2}{3}(h-k)-\frac{p}{2} \text {. }
$$

É fácil verificar que o ângulo $\theta$ satisfazendo esta equação é idêntico ao ângulo determinado por Arquimedes. Pois, na expressão acima,

$$
\frac{3 X}{2}=P V=k,
$$

de onde vem que

$$
a b=\frac{2}{3} h-\frac{p}{2}-\frac{2}{3} k=\frac{2}{3}(h-k)-\frac{p}{2} \text {. }
$$

Também

$$
b d^{2}=\frac{p}{4} \cdot a b \text {. }
$$

Segue que

$$
\left.\cot ^{2} d a b=\frac{a b^{2}}{b d^{2}}=\frac{4}{p}\left\{\frac{2}{3}(h-k)-\frac{p}{2}\right\} .\right]
$$

\section{Proposição 9}

Dado um sólido na forma de um segmento reto de um paraboloide de revolução cujo eixo $A M$ é maior que (3/4)p, mas tal que $A M: \frac{1}{2} p<15: 4$, e cuja gravidade específica está para a de um fluido em uma razão maior que $\left\{A M^{2}-\left(A M-\frac{3}{4} p\right)^{2}\right\}: A M^{2}$, então, se o sólido for colocado no fluido com seu eixo inclinado de um ângulo com a vertical, mas de forma que sua base esteja inteiramente abaixo da superfície, o sólido não retornará à posição na qual seu eixo esteja vertical e não permanecerá em qualquer posição, exceto aquela na qual seu eixo faz com a superfície do fluido um ângulo igual ao descrito na proposição anterior.

Tome am igual a $A M$ e tome $c$ sobre am tal que $a c=2 c m$. Meça co ao longo de ca igual a $\frac{1}{2} p$ e ar ao longo de ac tal que $a r=\frac{3}{2} a o$.

Seja $X+Y$ uma linha tal que 
(gravidade específica do sólido $):($ gravidade específica do fluido $)=\left\{a m^{2}-(X+Y)^{2}\right\}: a m^{2}$, e suponha $X=2 Y$.
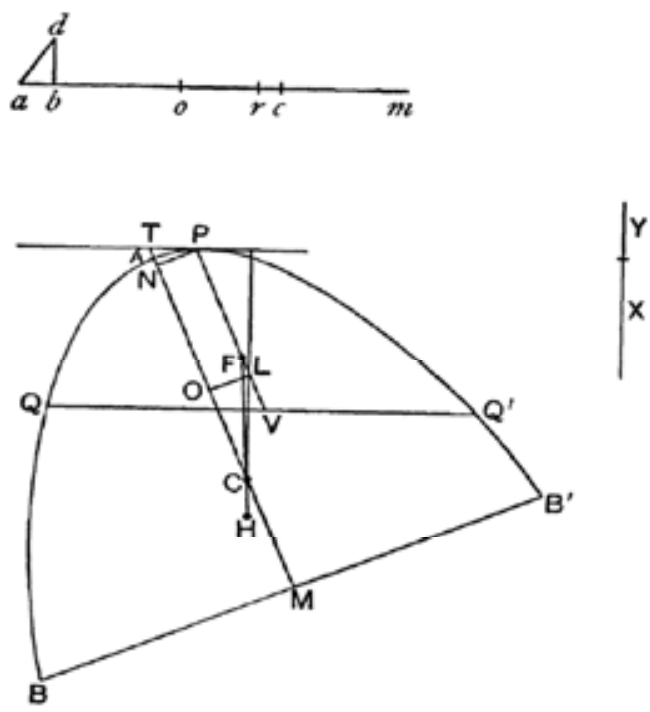

Agora,

$$
\begin{aligned}
a r & =\frac{3}{2} a o \\
& =\frac{3}{2}\left(\frac{2}{3} a m-\frac{1}{2} p\right) \\
& =A M-\frac{3}{4} p .
\end{aligned}
$$

Portanto, por hipótese,

$$
a m^{2}-a r^{2}: a m^{2}<\left\{a m^{2}-(X+Y)^{2}\right\}: a m^{2}
$$

de onde vem que

e, portanto,

$$
\begin{aligned}
X+Y & <a r, \\
X & <a o .
\end{aligned}
$$

Faça ob (medido ao longo de oa) igual a $X$, e trace $b d$ perpendicular a ba e com um comprimento tal que

$$
b d^{2}=\frac{1}{2} c o \cdot a b \text {. }
$$

Una $a d$.

Agora suponha o sólido colocado como na figura [acima] com seu eixo $A M$ inclinado em relação à vertical. Faça o plano através de $A M$, perpendicular à superfície do fluido, cortar o sólido na parábola $B A B^{\prime}$ e a superfície do fluido em $00^{\prime}$.

Seja $P T$ a tangente paralela a $Q Q^{\prime}, P V$ o diâmetro dividindo ao meio $Q Q^{\prime}$ (ou 0 eixo da porção do paraboloide acima da superfície), $P N$ a ordenada a partir de $P$.

I. Suponha o ângulo MTP maior que o ângulo dab. Seja $A M$ cortado como antes em $C$ e $O$ tal que $A C=2 C M$ e $O C=\frac{1}{2} p$, e da mesma forma $A M$ e am são igualmente divididos. Trace $O L$ perpendicular a $P V$.

Então temos, como na proposição anterior,

$$
P N^{2}: N T^{2}>d b^{2}: b a^{2}
$$


de onde vem que

e, portanto,

Segue que

$$
\begin{aligned}
c o: N T & >\frac{1}{2} c o: a b, \\
A N & <a b . \\
N O & >b o \\
& >X .
\end{aligned}
$$

Novamente, uma vez que a gravidade específica do sólido está para a do fluido assim como a porção imersa do sólido está para todo [0 sólido],

$$
\begin{array}{lc} 
& A M^{2}-(X+Y)^{2}: A M^{2}=A M^{2}-P V^{2}: A M^{2}, \\
& (X+Y)^{2}: A M^{2}=P V^{2}: A M^{2} . \\
\text { ou } & X+Y=P V . \\
\text { Isto é, } & P L(\text { ou } N O)>X \\
\text { E } & >\frac{2}{3} P V .
\end{array}
$$

Isto é,$$
\mathrm{E}
$$

de forma que

$P L>2 L V$.

Tome $F$ sobre $P V$ de forma que $P F=2 F V$. Então, $F$ é o centro de gravidade da porção do sólido sobre a superfície.

Também $C$ é 0 centro de gravidade de todo o sólido. Ligue $F C$ e o prolongue até $H, 0$ centro de gravidade da porção imersa.

Então, uma vez que $C O=\frac{1}{2} p, C L$ é perpendicular a $P T$ e à superfície do fluido; e a força atuando sobre a porção imersa do sólido age para cima ao longo da paralela a $C L$ através de $H$, enquanto o peso da parte restante do sólido age para baixo ao longo da paralela a $C L$ através de $F$.

Portanto, o sólido não ficará em repouso, mas vai girar na direção em que o ângulo MTP diminui.

II. Exatamente como na proposição anterior, provamos que, se 0 ângulo MTP for menor que o ângulo dab, 0 sólido não permanecerá em sua posição, mas girará na direção em que o ângulo MTP aumenta.

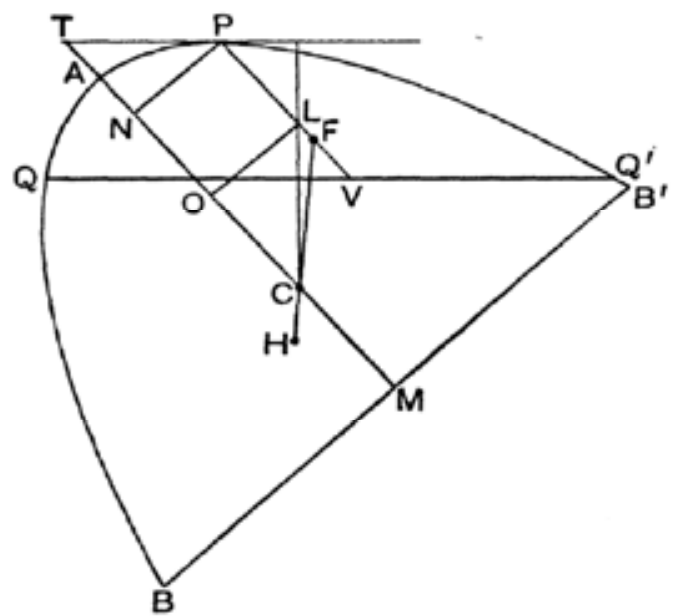

III. Se 0 ângulo MTP é igual ao ângulo dab, o sólido permanecerá em repouso nesta posição, porque $L$ e $F$ coincidirão, e todas as forças atuarão ao longo da linha CL. 


\section{Proposição 10}

Dado um sólido na forma de um segmento reto de um paraboloide de revolução no qual o eixo AM tem um comprimento tal que $A M: \frac{1}{2} p>15: 4$, e supondo o sólido colocado em um fluido com gravidade específica maior [que a sua] de forma que sua base esteja inteiramente sobre a superfície do fluido, investigar as posições de repouso.

\section{(Preliminar.)}

Suponha que o segmento de um paraboloide seja cortado por um plano através de seu eixo $A M$, no segmento parabólico $B A B_{1}$ do qual $B B_{1}$ é a base.

Divida $A M$ em $C$ de forma que $A C=2 C M$ e meça $C K$ ao longo de $C A$ tal que

$$
A M: C K=15: 4
$$

de onde vem, por hipótese, que $C K>\frac{1}{2} p$.

Suponha $C O$, medido ao longo de $C A$, igual a $\frac{1}{2} p$ e tome um ponto $R$ sobre $A M$ tal que $M R=\frac{3}{2} C O$.

Portanto,

$$
\begin{aligned}
A R & =A M-M R \\
& =\frac{3}{2}(A C-C O) \\
& =\frac{3}{2} A O .
\end{aligned}
$$

Una $B A$, trace $K A_{2}$ perpendicular a $A M$ encontrando $B A$ em $A_{2}$, divida ao meio $B A$ em $A_{3}$ e trace $A_{2} M_{2}$ e $A_{3} M_{3}$ paralelos a $A M$, encontrando $B M$ em $M_{2}$ e $M_{3}$, respectivamente.

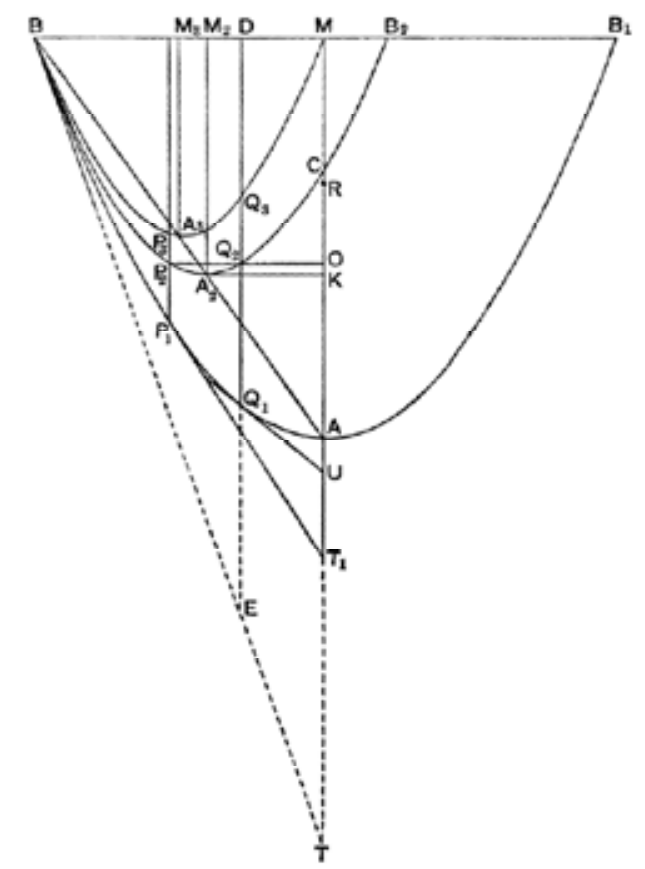

Tomando $A_{2} M_{2}$ e $A_{3} M_{3}$ como eixos, descreva segmentos parabólicos similares ao segmento $B A B_{1}$. (Segue, por semelhança de triângulos, que $B M$ será a base do segmento cujo eixo é $A_{3} M_{3}$ e $B B_{2}$ [será] a base daquele [segmento] cujo eixo é $A_{2} M_{2}$, onde $B B_{2}=2 B M_{2}$.) 
A parábola $B A_{2} B_{2}$ passará então através de $C$.

[Pois

$$
\begin{aligned}
B M_{2}: M_{2} M & =B M_{2}: A_{2} K \\
& =K M: A K \\
& =C M+C K: A C-C K \\
& =\left(\frac{1}{3}+\frac{4}{15}\right) A M:\left(\frac{2}{3}-\frac{4}{15}\right) A M \\
& =9: 6 \\
& =M A: A C
\end{aligned}
$$

Então vê-se que [o ponto] $C$ está sobre a parábola $B A_{2} B_{2}$ pela inversa da Prop. 4 da Quadratura da Parábola.]

Também, se uma perpendicular a $A M$ for traçada a partir de 0 , ela encontrará a parábola $B A_{2} B_{2}$ em dois pontos, em $\mathrm{O}_{2}$ e $P_{2}$. Trace $Q_{1} O_{2} O_{3} D$ através de $O_{2}$ paralelo a $A M$ encontrando as parábolas $B A B_{1}$ e $B A_{3} M$, respectivamente, em $Q_{1}$, e $Q_{3}$, e $B M$ em $D$; e seja $P_{1} P_{2} P_{3}$ a paralela correspondendo a $A M$ [passando] através de $P_{2}$. Faça as tangentes à parábola externa em $P_{1}$ e $Q_{1}$ encontrarem o prolongamento de $M A$ em $T_{1}$ e $U$, respectivamente.

Então, uma vez que os três segmentos parabólicos são similares e estão similarmente situados, com suas bases na mesma linha reta e tendo uma extremidade comum, e, uma vez que $Q_{1} Q_{2} O_{3} D$ é um diâmetro comum aos três segmentos, segue que:

$$
\begin{aligned}
& \text { Agora } \\
& \qquad \begin{aligned}
& Q_{1} Q_{2}: Q_{2} Q_{3}=\left(B_{2} B_{1}: B_{1} B\right) \cdot\left(B M: M B_{2}\right){ }^{6} \\
& B_{2} B_{1}: B_{1} B=M M_{2}: B M \\
&=2: 5, \\
& B M: M B_{2}=B M:\left(2 B M_{2}-B M\right) \\
&=5:(6-5), \\
&=5: 1 .
\end{aligned}
\end{aligned}
$$

Segue que

$$
Q_{1} Q_{2}: Q_{2} Q_{3}=2: 1
$$

6 [N. H.] Este resultado é assumido sem prova, sem dúvida como sendo uma dedução fácil a partir da Prop. 5 da Quadratura da Parábola. Ele pode ser estabelecido como segue.

Primeiro, uma vez que $A A_{2} A_{3} B$ é uma linha reta e $A N=A T$ em notação ordinária (onde $P T$ é a tangente em $P$ e $P N$ [é] a ordenada), segue, por semelhança de triângulos, que a tangente em $B$ à parábola externa é a tangente a cada uma das outras duas parábolas no mesmo ponto $B$.

Agora, pela proposiç̧ão citada, se o prolongamento de $D Q_{3} Q_{2} O_{1}$ encontra a tangente $B T$ em $E$,

$$
E Q_{3}: Q_{3} D=B D: D M \text {, }
$$

$$
\left.\begin{array}{l}
\text { de onde vem que } E Q_{3}: E D=B D: B M . \\
\text { Similarmente } E Q_{2}: E D=B D: B B_{2}, \\
\text { e } E Q_{1}: E D=B D: B B_{1} .
\end{array}\right\}
$$

As duas primeiras proposições são equivalentes a

$$
\begin{array}{ll}
E Q_{3}: E D=B D \cdot B B_{2}: B M \cdot B B_{2}, \\
\mathrm{e} \\
E Q_{2}: E D=B D \cdot B M: B M \cdot B B_{2} .
\end{array}
$$

Por subtração,

Similarmente,

$$
Q_{2} Q_{3}: E D=B D \cdot M B_{2}: B M \cdot B B_{2} .
$$

Segue que

$$
Q_{1} Q_{2}: E D=B D \cdot B_{2} B_{1}: B B_{2} \cdot B B_{1} \text {. }
$$

$$
Q_{1} Q_{2}: Q_{2} Q_{3}=\left(B_{2} B_{1}: B_{1} B\right) .\left(B M: M B_{2}\right) .
$$




$$
\left.\begin{array}{l}
\text { ou } Q_{1} Q_{2}=2 Q_{2} Q_{3} . \\
\text { Similarmente, } P_{1} P_{2}=2 P_{2} P_{3} .
\end{array}\right\}
$$

Também, uma vez que

$$
\begin{aligned}
M R & =\frac{3}{2} C O=\frac{3}{4} p, \\
A R & =A M-M R \\
& =A M-\frac{3}{4} p .
\end{aligned}
$$

\section{(Enunciado.)}

Se o segmento do paraboloide for colocado no fluido com sua base inteiramente acima da superfície, então (I.) se

(gravidade específica do sólido): (gravidade específica do fluido) $\nless A R^{2}: A M^{2}$

o sólido ficará em repouso na posição na qual seu eixo AM é vertical;

$$
\left[\nless(A M-3 p / 4)^{2}: A M^{2}\right] \text {, }
$$

(II.) se

(gravidade específica do sólido): (gravidade específica do fluido) $<A R^{2}: A M^{2}$

$$
\text { mas }>Q_{1} Q_{3}^{2}: A M^{2} \text {, }
$$

o sólido não ficará em repouso com sua base tocando a superfície do fluido em apenas um ponto, mas em uma posição tal que sua base não toque a superfície em qualquer ponto e seu eixo faça com a superfície um ângulo maior que U;

(III. a) se

(gravidade específica do sólido):(gravidade específica do fluido) $=Q_{1} Q_{3}{ }^{2}: A M^{2}$,

o sólido ficará em repouso e permanecerá na posição na qual a base toca a superfície do fluido em apenas um ponto e o eixo fará com a superfície um ângulo igual a U;

(III. b) se

(gravidade especifica do sólido): (gravidade especifica do fluido) $=P_{1} P_{3}^{2}: A M^{2}$,

o sólido ficará em repouso com sua base tocando a superfície do fluido em apenas um ponto e com seu eixo inclinado em relação à superfície em um ângulo igual a $T_{1}$;

(IV.) se

(gravidade específica do sólido):(gravidade específica do líquido $)>P_{1} P_{3}^{2}: A M^{2}$

$$
\text { mas }<Q_{1} Q_{3}^{2}: A M^{2} \text {, }
$$

o sólido permanecerá em repouso e ficará em uma posição com sua base mais submersa;

(V.) se

(gravidade específica do sólido): (gravidade especifica do fluido) $<P_{1} P_{3}^{2}: A M^{2}$,

o sólido permanecerá em repouso em uma posição na qual seu eixo esteja inclinado em relação à superfície do fluido em um ângulo menor que $T_{1}$, mas de modo que a base nem mesmo toque a superfície em um ponto.

\section{(Prova.)}

(I.) Um vez que $A M>\frac{3}{4} p$ e

(gravidade específica do sólido):(gravidade específica do fluido)

$$
\nless\left(A M-\frac{3}{4} p\right)^{2}: A M^{2} \text {, }
$$

segue, pela Prop. 4, que o sólido estará em equilíbrio estável com seu eixo na vertical.

(II.) Neste caso 
(gravidade específica do sólido):(gravidade específica do fluido) $<A R^{2}: A M^{2}$

$$
\text { mas }>Q_{1} Q_{3}{ }^{2}: A M^{2} \text {. }
$$

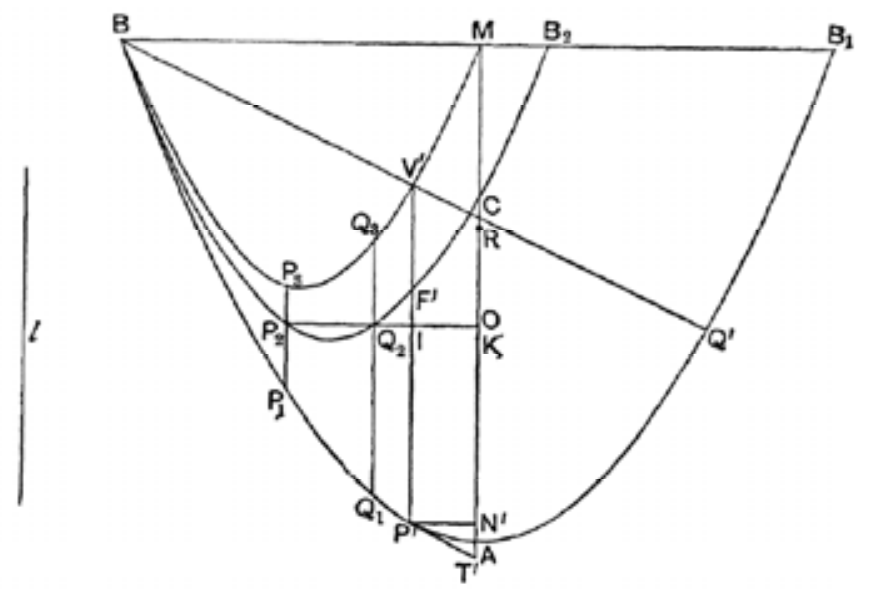

Suponha que a razão entre as gravidades específicas seja igual a

$$
l^{2}: A M^{2},
$$

de forma que $l<A R$ mas $>Q_{1} Q_{3}$.

Coloque $P^{\prime} V^{\prime}$ entre as duas parábolas $B A B_{1}$ e $B P_{3} Q_{3} M$ igual a / e paralelo a $A M^{7}$; e faça $P^{\prime} V^{\prime}$ encontrar a parábola intermediária em $F^{\prime}$.

7 [N. H.] Arquimedes não dá a solução deste problema, mas ela pode ser apresentada como segue.

Sejam $B R_{1} Q_{1}$ e $B R O_{2}$ dois segmentos parabólicos similares e similarmente situados, com suas bases na mesma linha reta e seja $B E$ a tangente comum em $B$.

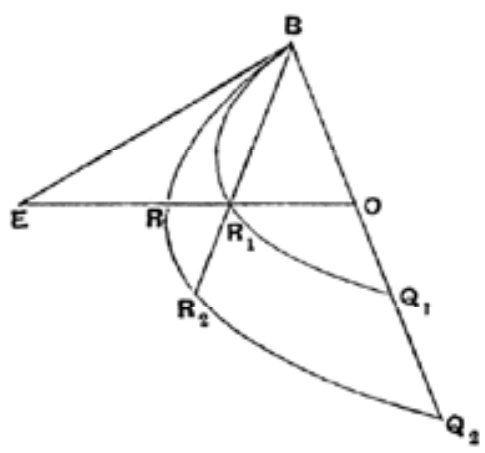

Suponha o problema resolvido, e faça $E R R_{1} 0$, paralelo aos eixos, encontrar as parábolas em $R$ e $R_{1}$, e $B O_{2}$ em 0 , fazendo o segmento $R R_{1}$ igual a $/$.

Então temos, como usual,

e

$$
\begin{aligned}
E R_{1}: E O & =B O: B Q_{1}, \\
& =B O \cdot B Q_{2}: B Q_{1} \cdot B Q_{2},
\end{aligned}
$$

$E R: E O=B O: B Q_{2}$

$$
=B O \cdot B Q_{1}: B Q_{1} \cdot B Q_{2} \text {. }
$$

Por subtração,

$$
R R_{1}: E O=B O \cdot Q_{1} Q_{2}: B Q_{1} \cdot B Q_{2} \text { ， }
$$

ou

$$
B O \cdot O E=l \cdot \frac{B Q_{1} \cdot B Q_{2}}{Q_{1} Q_{2}}, \text { o qual é conhecido. }
$$


Então, pela mesma prova como antes, obtemos

$$
P^{\prime} F^{\prime}=2 F^{\prime} V^{\prime} .
$$

Faça $P^{\prime} T^{\prime}$, a tangente em $P^{\prime}$ à parábola externa, encontrar $M A$ em $T^{\prime}$, e seja $P^{\prime} N^{\prime}$ a ordenada em $P^{\prime}$.

Una $B V^{\prime}$ e o prolongue para encontrar a parábola externa em $Q^{\prime}$. Faça $0 Q_{2} P_{2}$ encontrar $P^{\prime} V^{\prime}$ em $I$.

Agora, uma vez que, em dois segmentos parabólicos similares e similarmente situados, com bases $B M$ e $B B_{1}$ na mesma linha reta, $B V^{`}$ e $B Q^{\prime}$ são traçados fazendo o mesmo ângulo com as bases,

$$
\begin{aligned}
B V^{\prime}: B Q^{\prime} & =B M: B B_{1}{ }^{8} \\
& =1: 2, \\
B V^{\prime} & =V^{\prime} Q^{\prime} .
\end{aligned}
$$

de forma que

Suponha o segmento do paraboloide colocado no fluido, como descrito, com seu eixo inclinado de um ângulo em relação à vertical, e com sua base tocando a superfície somente em um ponto $B$. Seja o sólido cortado por um plano [passando] através do [seu] eixo e perpendicular à superfície do fluido, e faça o plano cortar o sólido no segmento parabólico $B A B^{\prime}$ e [cortar] o plano da superfície do fluido em $B O$.

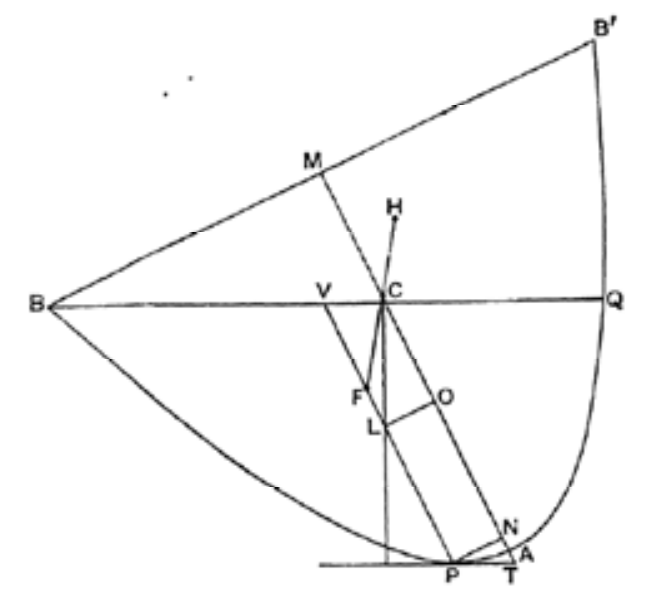

Tome os pontos $C$ e $O$ sobre $A M$ como descrito anteriormente. Trace a tangente paralela a $B O$ tocando a parábola em $P$ e encontrando $A M$ em $T$; e seja $P V$ o diâmetro dividindo ao meio $B O$ (isto é, o eixo da porção imersa do sólido).

Então,

$$
\begin{aligned}
l^{2}: A M^{2}=(\text { gravidade específica do sólido }):(\text { gravidade específica do fluido }) \\
=(\text { porção imersa }):(\text { todo o sólido }) \\
=P V^{2}: A M^{2},
\end{aligned}
$$

de onde vem que $\quad P^{\prime} V^{\prime}=l=P V$.

Então os segmentos nas duas figuras, isto é, $B P^{\prime} Q^{\prime}$ e $B P Q$, são iguais e similares.

Portanto, $\quad \angle P T N=\angle P^{\prime} T^{\prime} N^{\prime}$.

E a razão $B O: O E$ é conhecida. Portanto, $B O^{2}$, ou $O E^{2}$, pode ser encontrado e, portanto, $O$ [também pode ser encontrado].

${ }^{8}$ [N. H.] Para provar isso, suponha que, na figura da nota anterior, $B R_{1}$ seja prolongado para encontrar a parábola externa em $R_{2}$. Temos, como antes,

de onde vem que

$$
\begin{aligned}
& E R_{1}: E O=B O: B Q_{1}, \\
& E R: E O=B O: B Q_{2},
\end{aligned}
$$

$\mathrm{E}$, uma vez que $R_{1}$ é um ponto interno à parábola externa,

$E R: E R_{1}=B R_{1}: B R_{2}$, de uma forma semelhante.

Portanto,

$$
B Q_{1}: B Q_{2}=B R_{1}: B R_{2} \text {. }
$$


Também

$$
A T=A T^{\prime}, A N=A N^{\prime}, P N=P^{\prime} N^{\prime} .
$$

Agora, na primeira figura, $P^{\prime} I<2 I V^{\prime}$.

Portanto, se $O L$ for perpendicular a $P V$ na segunda figura,

$$
P L<2 L V \text {. }
$$

Tome $F$ sobre $L V$ de forma que $P F=2 F V$, isto é, de forma que $F$ seja o centro de gravidade da porção imersa do sólido. E seja $C$ o centro de gravidade de todo o sólido. Una $F C$ e o prolongue até $H$, o centro de gravidade da porção acima da superfície.

Agora, uma vez que $C O=\frac{1}{2} p, C L$ é perpendicular à tangente em $P$ e à superfície do fluido. Portanto, como antes, provamos que o sólido não ficará em repouso $\operatorname{com} B$ tocando a superfície, mas girará na direção em que 0 ângulo PTN aumenta.

Assim, na posição de repouso, o eixo $A M$ precisa fazer com a superfície do fluido um ângulo maior que 0 ângulo $U$ que a tangente em $Q_{1}$ faz com $A M$.

(III. a) Neste caso

(gravidade específica do sólido): (gravidade específica do fluido) $=Q_{1} Q_{3}{ }^{2}: A M^{2}$.

Seja o segmento do paraboloide colocado no fluido de forma que sua base não toque em lugar algum a superfície do fluido, e tal que seu eixo esteja inclinado de um ângulo com a vertical.

Faça o plano através de $A M$ perpendicular à superfície do fluido cortar o paraboloide na parábola $B A B^{\prime}$ e [cortar] o plano da superfície do fluido em $00^{\prime}$. Seja $P T$ a tangente paralela a $Q 0^{\prime}, P V$ o diâmetro dividindo ao meio $00^{\prime}$, e $P N$ a ordenada em $P$.

Divida $A M$ como antes em $C$ e 0 .
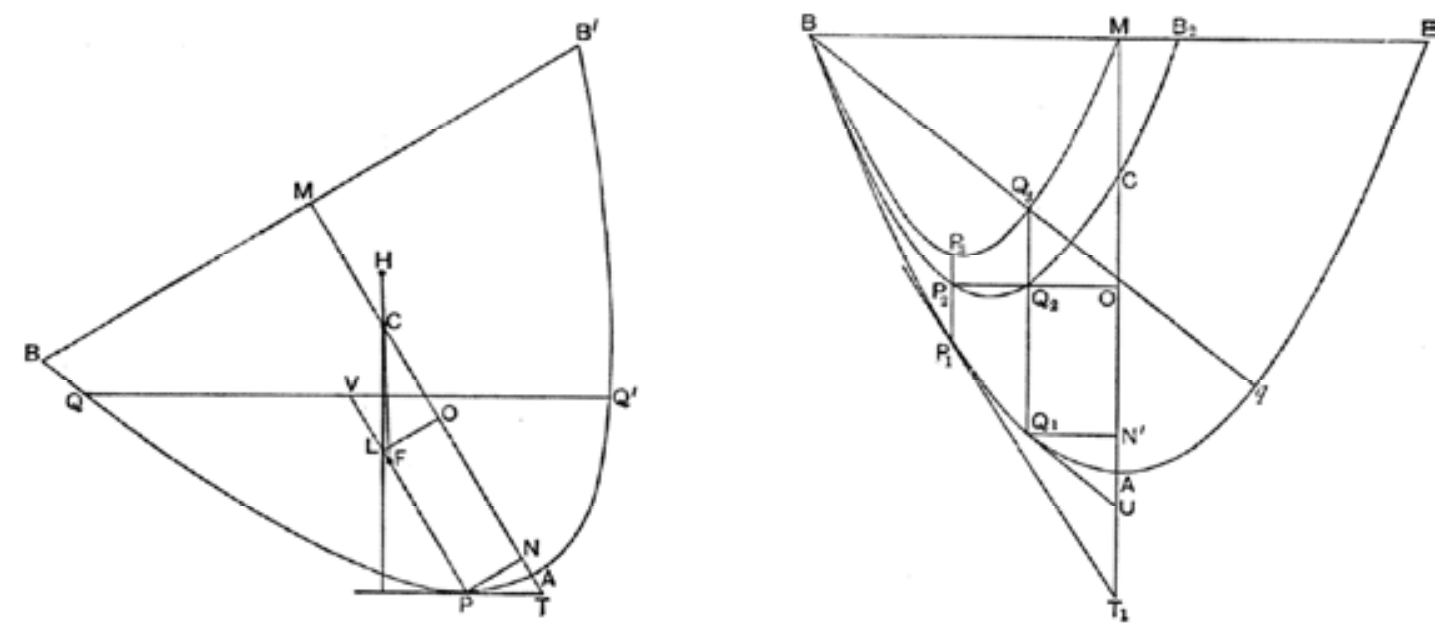

Na outra figura, seja $Q_{1} N^{\prime}$ a ordenada em $Q_{1}$. Una $B Q_{3}$ e o prolongue para encontrar a parábola externa em $q$. Então $B Q_{3}=Q_{3} p$, e a tangente $Q_{1} \cup$ é paralela a $B q$. Agora,

$$
\begin{aligned}
Q_{1} Q_{3}{ }^{2}: A M^{2}=(\text { gravidade específica do sólido }):(\text { gravidade específica do fluido }) \\
=(\text { porção imersa }):(\text { todo o sólido }) \\
=P V^{2}: A M^{2} .
\end{aligned}
$$

Portanto, $Q_{1} Q_{3}=P V$; e os segmentos $Q P Q^{\prime}$ e $B Q_{1} q$ do paraboloide são iguais em volume. E a base de um passa através de $B$, enquanto a base do outro passa através de 0 , um ponto mais próximo de $A$ do que $B$ está.

Segue que o ângulo entre $00^{\prime}$ e $B B^{\prime}$ é menor que o ângulo $B_{1} B q$. 
Portanto, $\angle U<\angle P T N$

de onde vem que

$A N^{\prime}>A N$,

e, portanto,

$N^{\prime} O\left(\right.$ ou $\left.Q_{1} Q_{2}\right)<P L$,

onde $O L$ é perpendicular a $P V$.

Segue, uma vez que $Q_{1} Q_{2}=2 Q_{2} Q_{3}$, que

$$
P L>2 L V \text {. }
$$

Portanto $F$, o centro de gravidade da porção imersa do sólido, está entre $P$ e $L$, enquanto, como antes, $C L$ é perpendicular à superfície do fluido.

Prolongando $F C$ até $H$, o centro de gravidade da porção do sólido acima da superfície, vemos que 0 sólido precisa girar na direção em que o ângulo PTN diminui, até que um ponto $B$ da base toque a superfície do fluido.

Quando este é o caso, teremos um segmento $B P Q$ igual e similar ao segmento $B Q_{1} q, 0$ ângulo PTN será igual ao ângulo $U$, e $A N$ será igual a $A N^{\prime}$.

Portanto, neste caso, $P L=2 L V$ e $F$ coincide com $L$, de forma que $F, C$ e $H$ estão todos em uma linha reta vertical.

Assim, o paraboloide permanecerá na posição na qual um ponto $B$ da base toca a superfície do fluido e 0 eixo faz com a superfície um ângulo igual a $U$.

(III. b) No caso onde

(gravidade específica do sólido): (gravidade específica do fluido) $=P_{1} P_{3}^{2}: A M^{2}$,

podemos, da mesma forma, provar que, se o sólido for colocado no fluido de forma que seu eixo esteja inclinado em relação à vertical e sua base não toque em nenhum lugar a superfície do fluido, o sólido se deslocará e ficará em repouso na posição na qual somente um ponto da base toca a superfície e 0 eixo estará inclinado em relação a ela de um ângulo igual a $T_{1}$ (na primeira figura da Prop. 10).

(IV.) Neste caso,

(gravidade específica do sólido): (gravidade específica do fluido) $>P_{1} P_{3}^{2}: A M^{2}$

$$
\text { mas }<Q_{1} Q_{3}^{2}: A M^{2} \text {. }
$$

Suponha que a razão seja igual a $l^{2}: A M^{2}$, de forma que / seja maior que $P_{1} P_{3}$, mas menor que $0_{1} Q_{3}$.

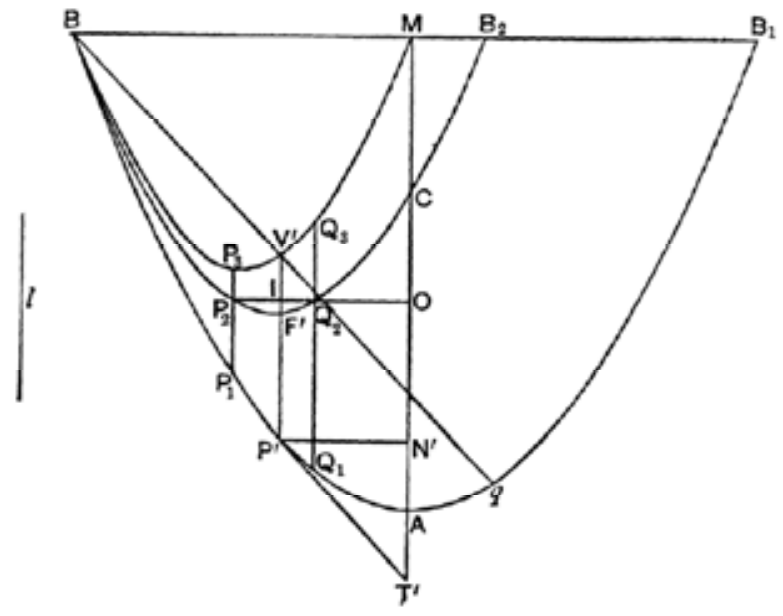

Coloque $P^{\prime} V^{\prime}$ entre as parábolas $B P_{1} Q_{1}$ e $B P_{3} Q_{3}$ de forma que $P^{\prime} V^{\prime}$ seja igual a / e paralelo a $A M$, e faça $P^{\prime} V^{\prime}$ encontrar a parábola intermediária em $F^{\prime}$ e $0 \mathrm{O}_{2} P_{2}$ em $/$.

Una $B V^{\prime}$ e o prolongue até encontrar a parábola externa em $q$.

Então, como antes, $B V^{\prime}=V^{\prime} q$, e assim a tangente $P^{\prime} T^{\prime}$ em $P^{\prime}$ é paralela a $B q$. Seja $P^{\prime} N^{\prime}$ a ordenada de $P^{\prime}$.

1. Agora seja o segmento colocado no fluido, primeiro com seu eixo tão inclinado em relação à vertical que sua base não toque em nenhum lugar a superfície do fluido. 


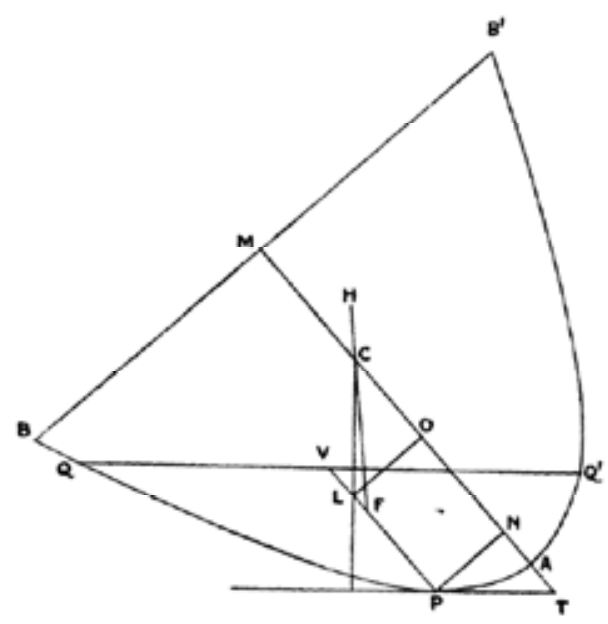

Faça o plano através de $A M$ perpendicular à superfície do fluido cortar o paraboloide na parábola $B A B^{\prime}$ e [cortar] o plano da superfície do fluido em $Q Q^{\prime}$. Seja $P T$ a tangente paralela a $Q Q^{\prime}$, [e seja] $P V$ o diâmetro dividindo ao meio $Q Q^{\prime}$. Divida $A M$ em $C$ e $O$ como antes, e trace $O L$ perpendicular a $P V$.

Então, como antes, temos $P V=I=P^{\prime} V^{\prime}$.

Portanto, os segmentos $B P^{\prime} q$ e $Q P Q^{\prime}$ do paraboloide são iguais em volume; e segue que o ângulo entre $00^{\prime}$ e $B B^{\prime}$ é menor que o ângulo $B_{1} B q$.

$$
\begin{array}{lc}
\text { Portanto, } & \angle P^{\prime} T^{\prime} N^{\prime}<\angle P T N, \\
\text { e então } & A N^{\prime}>A N, \\
\text { de forma que } & N O>N^{\prime} O, \\
\text { isto é, } & P L>P^{\prime} I \\
& >P^{\prime} F^{\prime}, \text { a fortiori. }
\end{array}
$$

Portanto, $P L>2 L V$, de modo que $F, 0$ centro de gravidade da porção imersa do sólido, está entre $L$ e $P$, enquanto $C L$ é perpendicular à superfície do fluido.

Se, então, prolongarmos $F C$ até $H$, o centro de gravidade da porção do sólido acima da superfície, provamos que 0 sólido não ficará em repouso, mas girará na direção em que o ângulo PTN diminui.

2. A seguir, seja o paraboloide colocado no fluido de forma que sua base toque a superfície do fluido somente em um ponto $B$, e proceda à construção como anteriormente.

Então $P V=P^{\prime} V^{\prime}$ e os segmentos $B P 0$ e $B P^{\prime} q$ são iguais e similares, de forma que

$$
\angle P T N=\angle P^{\prime} T^{\prime} N^{\prime} \text {. }
$$

Segue que

$$
A N=A N^{\prime}, N O=N^{\prime} O \text {, }
$$

e, portanto, $P^{\prime} I=P L$ ，

de onde vem que

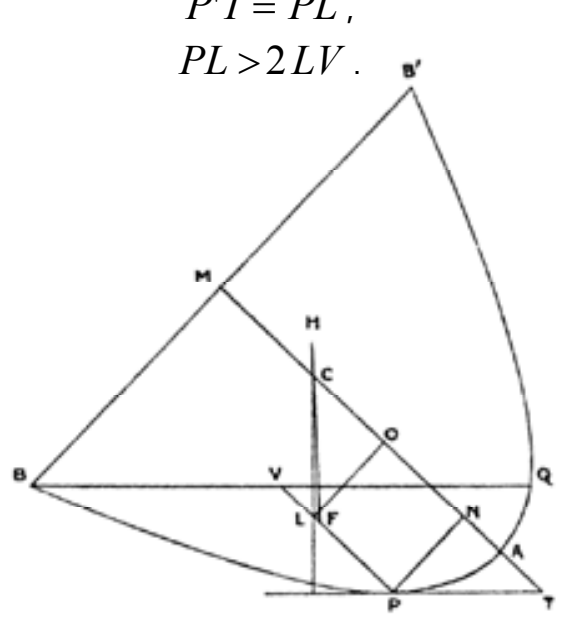


Portanto, $F$ novamente se encontra entre $P$ e $L$, e, como antes, o paraboloide girará na direção em que 0 ângulo PTN diminui, isto é, de forma que a base será mais submersa.

(V.) Neste caso

(gravidade específica do sólido): (gravidade específica do fluido) $<P_{1} P_{3}^{2}: A M^{2}$.

Se então a razão é igual a $l^{2}: A M^{2}, l<P_{1} P_{3}$. Coloque $P^{\prime} V^{\prime}$ entre as parábolas $B P_{1} Q_{1}$ e $B P_{3} O_{3}$ igual em comprimento a / e paralelo a $A M$. Faça $P^{\prime} V^{\prime}$ encontrar a parábola intermediária em $F^{\prime}$ e $O P_{2}$ em $/$.

Una $B V^{\prime}$ e o prolongue até encontrar a parábola externa em $q$. Então, como antes, $B V^{\prime}=V^{\prime} q$ e a tangente $P^{\prime} T^{\prime}$ é paralela a $B q$.

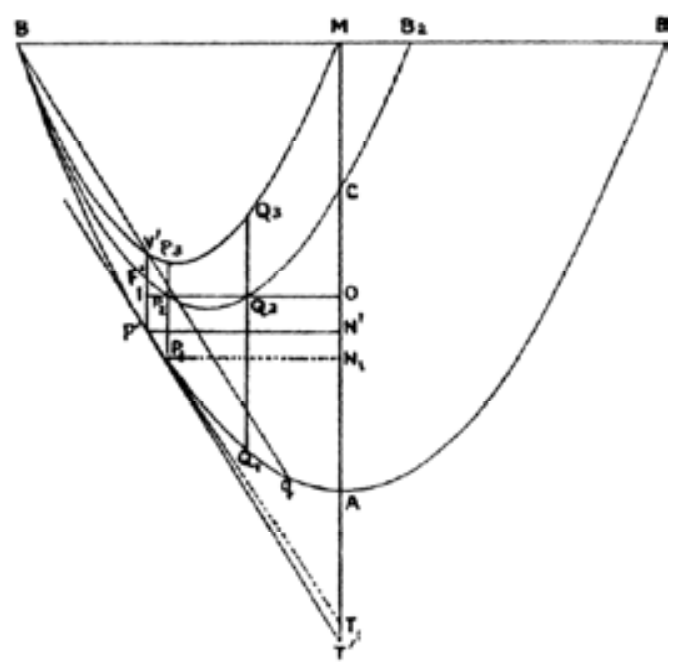

1. Seja o paraboloide colocado no fluido de forma que sua base toque a superfície somente em um ponto.

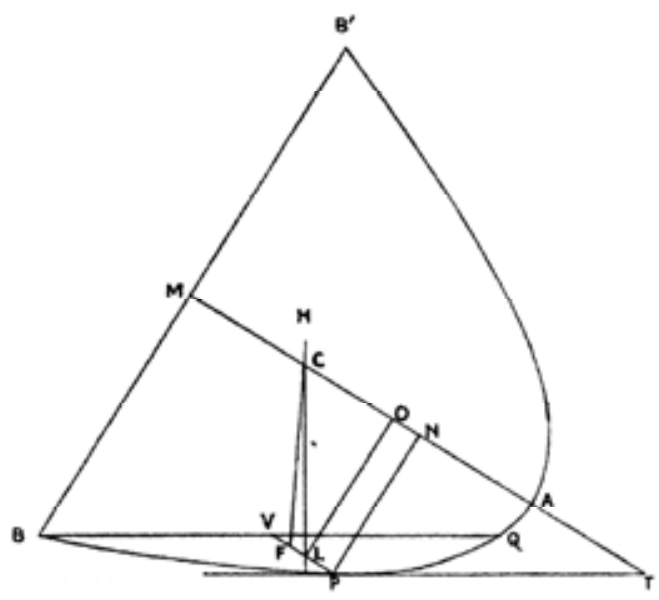

Faça o plano através de $A M$ perpendicular à superfície do fluido cortar o paraboloide na seção parabólica $B A B^{\prime}$ e [cortar] o plano da superfície do fluido em $B O$.

Fazendo a construção usual, encontramos

$$
P V=l=P^{\prime} V^{\prime},
$$

e os segmentos $B P Q$ e $B P_{1} q$ são iguais e similares.

Portanto, $\quad \angle P T N=\angle P^{\prime} T^{\prime} N^{\prime}$ ， 
e

Portanto,

de onde segue que

$$
A N=A N^{\prime}, N^{\prime} O=N O .
$$

$$
P L=P^{\prime} I \text {, }
$$$$
P L<2 L V
$$

Portanto $F$, o centro de gravidade da porção imersa do sólido, encontra-se entre $L$ e $V$, enquanto $C L$ é perpendicular à superfície do fluido.

Prolongando FC até $H$, o centro de gravidade da porção acima da superfície, provamos, da forma usual, que não haverá repouso, mas o sólido girará na direção em que o ângulo PTN aumenta, de forma que a base não tocará a superfície em qualquer lugar.

2. 0 sólido, entretanto, ficará em repouso em uma posição onde seu eixo faz com a superfície do fluido um ângulo menor que $T_{1}$.

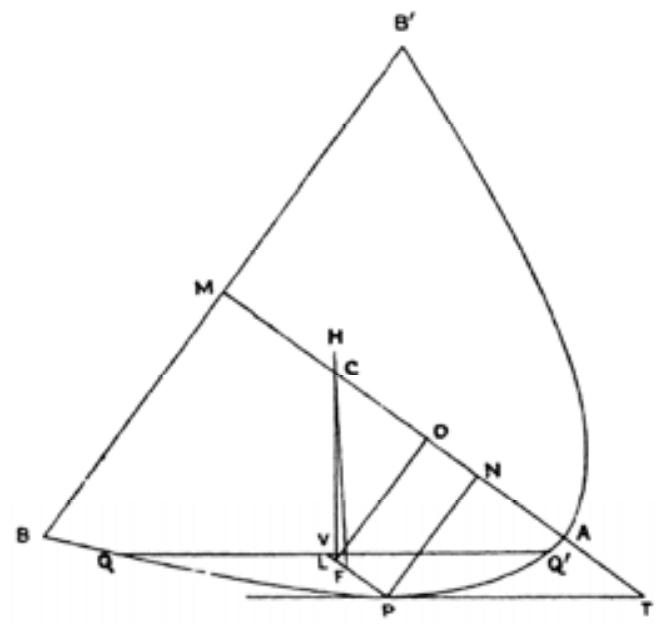

Seja [o sólido] colocado de forma que o ângulo PTN não é menor que $T_{1}$.

Então, com a mesma construção de antes, $P V=l=P^{\prime} V^{\prime}$.

$E$, uma vez que

$\angle T \nless \angle T_{1}$,

$$
A N \ngtr A N_{1} \text {, }
$$

e, portanto, $N O \nless N_{1} O$, onde $P_{1} N_{1}$ é a ordenada de $P_{1}$.
Portanto,
$P L \nless P_{1} P_{2}$.
Mas
$P_{1} P_{2}>P^{\prime} F^{\prime}$.
Portanto,

$$
P L>\frac{2}{3} P V
$$

de forma que $F$, o centro de gravidade da porção imersa do sólido, encontra-se entre $P$ e $L$.

Assim, o sólido girará na direção em que o ângulo PTN diminui, até que este ângulo fique menor que $T_{1}$.

[Como antes, se $x$ e $x^{\prime}$ forem as distâncias a partir de $T$ das projeções ortogonais de $C$ e $F$, respectivamente, sobre TP, temos

$$
x^{\prime}-x=\cos \theta\left\{\frac{p}{4}\left(\cot ^{2} \theta+2\right)-\frac{2}{3}(h-k)\right\}
$$

onde $h=A M, k=P V$. 
Também, se a base $B B^{\prime}$ toca a superfície do fluido em um ponto $B$, temos ainda, como na nota acompanhando a Prop. 6,

$$
\begin{array}{r}
\sqrt{p h}=\sqrt{p k}+\frac{p}{2} \cot \theta \ldots \ldots \\
h-k=\sqrt{p h} \cot \theta-\frac{p}{4} \cot ^{2} \theta
\end{array}
$$

Portanto, para encontrar a relação entre $h$ e 0 ângulo $\theta$ no qual o eixo do paraboloide está inclinado em relação à superfície do fluido em uma posição de equilíbrio, $\operatorname{com} B$ apenas tocando a superfície, eliminamos $k$ e igualamos a zero a expressão em (1); então

$$
\frac{p}{4}\left(\cot ^{2} \theta+2\right)-\frac{2}{3}\left(\sqrt{p h} \cot \theta-\frac{p}{4} \cot ^{2} \theta\right)=0,
$$

ou

$$
5 p \cot ^{2} \theta-8 \sqrt{p h} \cot \theta+6 p=0
$$

Os dois valores de $\theta$ são dados pelas equações

$$
5 \sqrt{p} \cot \theta=4 \sqrt{h} \pm \sqrt{16 h-30 p}
$$

0 sinal inferior corresponde, na proposição de Arquimedes, ao ângulo $U$ e 0 sinal superior ao ângulo $T_{1}$, como pode ser verificado assim.

Na primeira figura de Arquimedes (primeira figura da Prop. 10) temos

$$
\begin{gathered}
A K=\frac{2}{5} h, \\
M_{2} D^{2}=\frac{3}{5} p \cdot O K=\frac{3}{5} p\left(\frac{2}{3} h-\frac{2}{5} h-\frac{1}{2} p\right) \\
=\frac{3 p}{5}\left(\frac{4 h}{15}-\frac{p}{2}\right) .
\end{gathered}
$$

Se $P_{1} P_{2} P_{3}$ encontra $B M$ em $D^{\prime}$, segue que

$$
\begin{aligned}
& \left.\begin{array}{l}
M_{3} D \\
M_{3} D^{\prime}
\end{array}\right\}=M_{2} D \pm M_{3} M_{2} \\
& =\sqrt{\frac{3 p}{5}\left(\frac{4 h}{15}-\frac{p}{2}\right)} \pm \frac{1}{10} \sqrt{p h}
\end{aligned}
$$

e

$$
\begin{aligned}
& \left.\begin{array}{l}
M D^{\prime} \\
M D^{\prime}
\end{array}\right\}=M M_{2} \mp M_{2} D \\
& =\frac{2}{5} \sqrt{p h} \mp \sqrt{\frac{3 p}{5}\left(\frac{4 h}{15}-\frac{p}{2}\right)} .
\end{aligned}
$$

Agora, da propriedade da parábola,

de forma que

$$
\begin{gathered}
\cot U=2 \frac{M D}{p} \\
\cot T_{1}=2 \frac{M D^{\prime}}{p} \\
\frac{p}{2} \cot \left\{\begin{array}{l}
U \\
T_{1}
\end{array}\right\}=\frac{2}{5} \sqrt{p h} \mp \sqrt{\frac{3 p}{5}\left(\frac{4 h}{15}-\frac{p}{2}\right)},
\end{gathered}
$$




$$
5 \sqrt{p} \cot \left\{\begin{array}{l}
U \\
T_{1}
\end{array}\right\}=4 \sqrt{h} \mp \sqrt{16 h-30 p},
$$

o que concorda com o resultado (5) acima.

Para encontrar a razão correspondente das gravidades específicas, ou $k^{2} / h^{2}$, temos que usar as equações (2) e (5) e expressar $k$ em termos de $h$ e $p$.

A equação (2) fornece, pela substituição nela do valor de cot $\theta$ contido em (5),

de onde obtemos, elevando ao quadrado,

$$
\begin{aligned}
\sqrt{k} & =\sqrt{h}-\frac{1}{10}(4 \sqrt{h} \pm \sqrt{16 h-30 p}) \\
& =\frac{3}{5} \sqrt{h} \mp \frac{1}{10} \sqrt{16 h-30 p},
\end{aligned}
$$

$$
k=\frac{13}{25} h-\frac{3}{10} p \mp \frac{3}{25} \sqrt{h(16 h-30 p)}
$$

0 sinal inferior corresponde ao ângulo $U$ e 0 superior ao ângulo $T_{1}$, e, a fim de verificar os resultados de Arquimedes, temos que mostrar simplesmente que os dois valores de $k$ são iguais a $Q_{1} O_{3}$ e $P_{1} P_{3}$, respectivamente.

Agora, é fácil ver que

$$
\begin{aligned}
& Q_{1} Q_{3}=\frac{h}{2}-\frac{M D^{2}}{p}+2 \frac{M_{3} D^{2}}{p}, \\
& P_{1} P_{3}=\frac{h}{2}-\frac{M D^{\prime 2}}{p}+2 \frac{M_{3} D^{\prime 2}}{p} .
\end{aligned}
$$

Portanto, usando os valores de $M D, M D^{\prime}, M_{3} D$ e $M_{3} D^{\prime}$ determinados acima, temos

$$
\begin{aligned}
& \left.\begin{array}{l}
Q_{1} Q_{3} \\
P_{1} P_{3}
\end{array}\right\}=\frac{h}{2}+\frac{3}{5}\left(\frac{4 h}{15}-\frac{p}{2}\right)-\frac{7 h}{50} \pm \frac{6}{5} \sqrt{\frac{3 h}{5}\left(\frac{4 h}{15}-\frac{p}{2}\right)} \\
& =\frac{13}{25} h-\frac{3}{10} p \pm \frac{3}{25} \sqrt{h(16 h-30 p)},
\end{aligned}
$$

que são os valores de $k$ dados em (6) acima.]

\section{Notas e referências bibliográficas}

André Koch Torres Assis é doutor em Física pela Universidade Estadual de Campinas - UNICAMP e professor associado da Universidade Estadual de Campinas - UNICAMP. E-mail: assis@ifi.unicamp.br

Nivaldo Benedito Ferreira Campos é doutor em Engenharia Mecânica pela Universidade Estadual de Campinas - UNICAMP e professor da Universidade Estadual de Campinas - UNICAMP. E-mail: nivaldo.campos@unifesp.br

1 ASSIS, A. K. T. Sobre os corpos flutuantes, Tradução comentada de um texto de Arquimedes. Revista da Sociedade Brasileira de História da Ciência, v. 16 , p. 69-80, 1996.

2 ARQUIMEDES. On floating bodies (book II). In: HEATH, T. L. (Org.). Archimedes. Nova lorque: Dover, 2002. p. 263-300. 